\title{
ORIGINAL
}

\section{FRECUENCIA DEL ESOFAGO DE BARRETT POR REFLUJO GASTROESOFAGICO EN LA UNIVERSIDAD CENTRAL DE VENEZUELA.}

\author{
FRECUENCY OF BARRET'S ESOPHAGUS FOR GASTROESOPHAGEAL \\ REFLUX IN THE CENTRAL UNIVERSITY OF VENEZUELA.
}

'Dra. Dilia Díaz Arreaza Jefe de la sección de patología gastrointestinal y hepática "Dr. Pedro Grases" del Instituto

Anatomopatológico “Dr. José A O’Daly". Universidad Central de Venezuela. Caracas. Venezuela.

Correspondencia a:

Nombre: Dra. Dilia Díaz Arreaza

Correo electrónico: diliacda@

gmail.com

Telf. y celular: +584147910032

Palabras clave: Biopsia, esófago, esofagitis por reflujo, Esófago de Barret, cáncer.

Keywords: Biopsy, esophagus, reflux esophagitis, Barrett esophagus, cancer

Procedencia y arbitraje: no comisionado, sometido a arbitraje externo.

Recibido para publicación 04 de octubre de 2017 Aceptado para publicación 14 de julio de 2018

Citar como:

Rev Cient Cienc Med

2018;21(1):21-29

\author{
Cuauro Ruíz Gabriela Díaz Arreaza Dilia Ruíz Gómez María Elena Merheb Luttinger \\ Michelle ${ }^{1}$ Malpica Pittol Alisson ${ }^{1}$
}

\section{RESUMEN}

Introducción: Enfermedad por reflujo se desarrolla cuando el contenido gástrico provoca síntomas y/o complicaciones esofágicas y extra esofágicas. Las esofágicas incluyen el reflujo, esofagitis de reflujo y el esófago de Barrett. Esta última premaligna, caracterizada por una metaplasia intestinal especializada cuya importancia es el conocimiento de la displasia por su evolución a cáncer. Trabajos anteriores determinaron, displasia en esófago de Barrett $33,3 \%$; prevalencia de enfermedad por reflujo no erosiva $82,70 \%$ y erosiva $17,30 \%$; de esofagitis con hallazgo endoscópico e histológico $84,37 \%$.

Objetivo: Evaluar la prevalencia del esófago de Barrett por reflujo y esofagitis por reflujo en el Instituto Anatomopatológico de la Universidad Central de Venezuela.

Método: Se realizó un estudio descriptivo, retrospectivo. Se estudiaron las muestras endoscópicas de biopsias esofágicas que ingresaron en el período 2005 al 20I5, con diagnóstico de reflujo y esofagitis por reflujo. Se evaluó la ausencia o presencia de displasia en el esófago de Barrett, con su gradación correspondiente. Se utilizó un análisis descriptivo presentado en forma de frecuencias absolutas y relativas.

Resultados: $35,85 \%$ reflujo y $64,12 \%$ esofagitis por reflujo, ambas con predominio en mujeres y edad promedio de 55,2 y $53, I$ años respectivamente. Dieciocho casos (8\%) con esófago de Barrett en relación a enfermedad por reflujo. Displasia de bajo grado $5,55 \%$, indefinido para displasia $50 \%$ y negativo para displasia $44,44 \%$.

Conclusiones: La prevalencia de esófago de Barrett asociado a enfermedad por reflujo gastroesofágico fue del $8 \%$. La mayor frecuencia fue en el sexo femenino con $66,66 \%$, y las edades comprendidas entre 30 y 78 años con una media de 59,25 años.

\section{ABSTRACT}

Introduction: Reflux disease develops when gastric content causes esophageal and extra esophageal symptoms and/or complications. The esophageal complications include reflux, reflux esophagitis and Barrett's esophagus. The last is a pre-malignant condition characterized by a specialized intestinal metaphase whose importance lies in the knowledge of dysplasia due to its evolution to cancer. Previous investigations determined the prevalence of: dysplasia in Barrett's esophagus 33,3\%; non erosive reflux disease $82,70 \%$ and erosive $17,30 \%$; esophagitis with endoscopic and morphologic findings $84,37 \%$.

Objective: To evaluate the prevalence of Barrett's esophagus in patients with reflux and reflux esophagitis in the Anatomopathologic Institute of Universidad Central de Venezuela.

Method: A descriptive and retrospective study was conducted. There were evaluated the esophageal endoscopic biopsies between 2005-2015, with diagnosis of reflux and reflux esophagitis. The presence or absence of dysplasia was reviewed in the Barrett's esophagus cases, with the respective grade.Absolute and relative frequencies were obtained by a descriptive analysis.

Results: $35,85 \%$ of cases were reflux and $64,12 \%$ were reflux esophagitis, both with women predominance and the average age was 55,2 and 53, I years respectively. Eighteen cases (8\%) with Barrett's esophagus were related to reflux disease. Low grade dysplasia represented 5,55\%, indefinite for dysplasia $50 \%$ and negative for dysplasia $44,44 \%$. Conclusions:The prevalence of Barrett's esophagus associated with gastroesophageal reflux disease was $8 \%$. The highest frequency was in the female sex with $66,66 \%$, and the ages between 30 and 78 years with an average of 59,25 years. 
INTRODUCCIÓN

a enfermedad por reflujo es una condición que se de- sarrolla cuando el contenido gástrico provoca síntomas como acidez y regurgitaciones, y/o complicaciones esofágicas y extraesofágicas ${ }^{1,2}$. Entre las complicaciones esofágicas encontramos el reflujo, que histológicamente, se caracteriza por un epitelio plano estratificado no queratinizado con acantosis, papilomatosis en dos tercios de la superficie e hiperplasia de la capa basal en más de un $20 \%$ del epitelio. Si a estos cambios, se le asocia exocitosis de polimorfonucleares neutrófilos y eosinófilos, se diagnostica como esofagitis por reflujo ${ }^{3,4}$.

Para el diagnóstico de los trastornos inflamatorios en el esófago, se necesitan exámenes endoscópicos e histopatológicos principalmente cuando se trata de reflujo, esofagitis por reflujo y esófago de Barrett ${ }^{5}$.

Es bien conocido que en condiciones normales la unión esófagogástrica es simétrica y abrupta, en la cual histológicamente se delimita muy bien el epitelio plano estratificado no queratinizado del cilíndrico simple correspondiente al glandular. Si se observa presencia de mucosa gástrica por debajo del epitelio esofágico, estamos en presencia de una metaplasia columnar6. También es importante reconocer el epitelio híbrido, en el cual se evidencia de manera transicional el cambio entre el epitelio esofágico y gástrico, observándose en la parte inferior del epitelio escamoso células columnares mucosas, lo cual representa un cambio adaptativo y se ha asociado como precursor del esófago de Barre$\mathrm{tt}$, que es la presencia de células caliciformes en la mucosa glandular 5 .

El esófago de Barrett es una condición premaligna que se define como la sustitución de la mucosa escamosa estratificada esofágica normal por epitelio columnar metaplásico especializado, ya que tiene la presencia de células caliciformes (ver Figura 1) ${ }^{7,8}$. La mayoría de las evidencias clínicas y experimentales, indican que esta metaplasia o cambio de

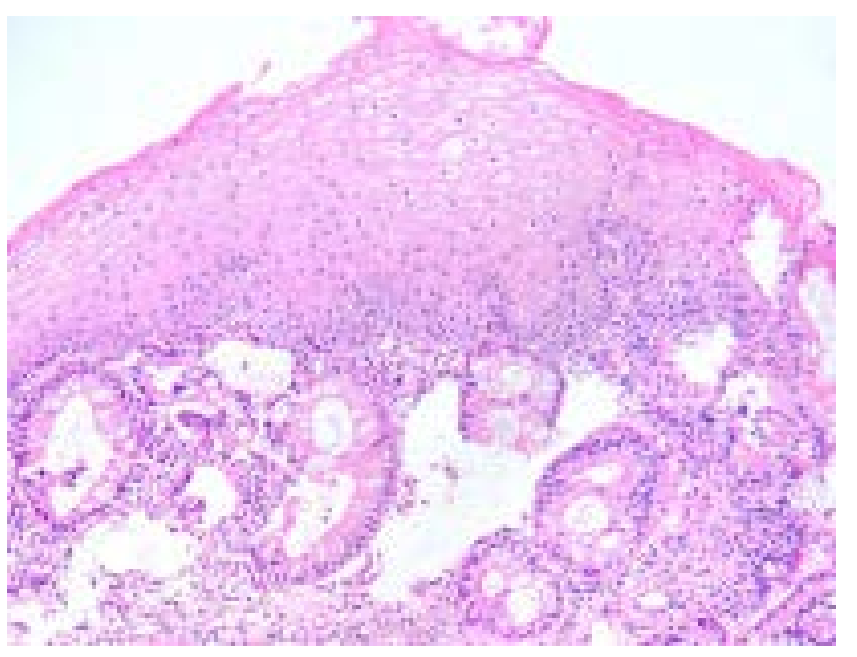

Figura 1. Esófago de Barrett. Coloración H-E. 200X epitelio es adquirida y es el resultado de un reflujo gastroesofágico prolongado que conlleva a inflamación y posterior ulceración del epitelio de revestimiento y la subsecuente reepitelización por células madres in situ, que a un $\mathrm{pH}$ anormalmente bajo, secundario al reflujo, se diferenciaran a un epitelio columnar metaplásico como un proceso adaptati$v^{4}$. El diagnóstico debe cumplir dos criterios, endoscópicos e histológicos. El primero de ellos es la observación del desplazamiento de la unión escamocolumnar en sentido proximal en el esófago distal, en la que se evidencia un epitelio columnar de color rojizo brillante de aspecto aterciopelado, en yuxtaposición con un epitelio plano estratificado de color blanquecino, por encima de la línea $Z$, correspondiente a la unión escamocolumnar ${ }^{9}$. El segundo criterio corresponde a los hallazgos histológicos antes mencionados ${ }^{10}$. Es importante el reconocimiento de displasia en el esófago de Barrett ya que este puede evolucionar a cáncer y es por ello que para el reporte diagnóstico existe las categorías de displasia según el consenso de 1988, se clasifican como negativo para displasia, indefinido para displasia, con displasia de bajo y con displasia de alto grado y las mismas deben ser especificadas en cada caso ${ }^{5,11,12}$.

Trabajos anteriores como el realizado por García, en el Instituto Anatomopatológico de la Universidad Central de Venezuela durante los años 1995 al 1999, se estudiaron 266 casos con esofagitis por reflujo y esófago de Barrett, determinando presencia de displasia en esófago de Barrett en el $33,3 \%{ }^{13}$. Otra investigación realizada en el 2010 por Poleo, en la que hace referencia a la prevalencia de enfermedad por reflujo gastroesofágico en dos muestras de población venezolana resultó con una prevalencia de enfermedad por reflujo no erosiva en $82,70 \%$ y la enfermedad por reflujo gastroesofágico erosiva en $17,30 \%{ }^{14}$. Un trabajo más reciente del 2011 de Cedeño, et al. en el que estudiaron sobre esofagitis por reflujo con diagnóstico histológico y su relación con variables clínicas y endoscópicas, la prevalencia de esofagitis $A$ y $B$, según la Clasificación de Los Angeles, con hallazgo endoscópico e histológico fue de $84,37 \%{ }^{15}$. Por estos estudios previos, nuestro objetivo fue evaluar la prevalencia del esófago de Barrett en pacientes con reflujo y esofagitis por reflujo en el Instituto Anatomopatológico “Dr. José A O’Daly” de la Universidad Central de Venezuela.

\section{MATERIALES Y MÉTODOS}

Se realizó un estudio descriptivo, retrospectivo. Realizado en la sección de patología gastrointestinal y hepática “Dr. Pedro Grases" del Instituto Anatomopatológico “Dr. José A O’Daly" de la Universidad Central de Venezuela, donde se estudiaron 702 muestras endoscópicas de biopsias esofágicas que ingresaron durante el periodo 2005 al 2015. Se revisaron los informes anatomopatológicos, incluyendo en el 
estudio todas aquellas muestras con diagnóstico de reflujo y esofagitis por reflujo que contaban con láminas histológicas y/o bloques de parafina en el archivo para su revisión. Además se revisaron las boletas de solicitud de biopsia de los 702 pacientes, donde se obtuvo la información clínica respecto al sexo y edad del paciente. Fueron excluidas aquellas muestras que no tenían archivo y aquellas donde no se determinó el género y/o la edad. Cumplieron con los criterios de inclusión 223 biopsias cuyas láminas fueron revisadas en un Microscopio Olympus ${ }^{\circledR}$ Cx 31 por dos patólogos especialistas en el área, los cuales corroboraron los diagnósticos. Se evaluó la ausencia o presencia de displasia en el esófago de Barrett, con su gradación correspondiente. La recolección de los datos se llevó a cabo con una tabla Excel $^{\oplus}$, donde se vacío la información correspondiente a: número de caso, edad del paciente, sexo del paciente, reflujo, esofagitis por reflujo, esófago de Barrett, negativo para displasia, indefinido para displasia, displasia de bajo grado, displasia de alto grado. Se obtuvo el número de casos con reflujo y esofagitis por reflujo con su respectiva proporción, determinando el promedio de edad y la distribución por sexo. Asociando cada una de estas patologías con el esófago de Barrett y obteniendo de esta manera la frecuencia que cuantifica la proporción de individuos de una población que padecen una enfermedad en un periodo de tiempo determinado. Todos los pacientes firmaron su consentimiento informado para la realización de la biopsia, misma que estaba adjunta en la historia clínica; todos los datos se manejaron en base a un código alfanumérico, en respeto a la autonomía del paciente, sin interferencia en ningún proceso que involucre su salud ni su bienestar social; en cumplimiento a las normas éticas vigentes.

\section{RESULTADOS}

Se revisaron 702 biopsias endoscópicas de esófago en 10 años de los cuales $223(31,76 \%)$ casos cumplieron con los criterios de inclusión. Del total de los pacientes que ingresaron al estudio; 80 (35,85\%) tenían reflujo y $143(64,12 \%)$ esofagitis por reflujo. La edad de presentación en el reflujo, fue en promedio de 55,21 años, siendo el de menor edad un paciente de 1 año y la mayor edad 81 años. La distribución por sexo fue mayor en el femenino con 42 pacientes (52,5\%). Nueve casos estaban asociados a esófago de Barrett (ver Figura 2), siete mujeres y dos hombres, con edades comprendidas entre 30 y 78 años y una media de 57 años. Se observó displasia de bajo grado en un paciente (ver Figura 3), cuatro entraron en la categoría indefinido para displasia y cuatro fueron negativos para la misma. La edad promedio de presentación en la esofagitis por reflujo, fue 53,14 años, el paciente de menor edad tenía 1 año y el mayor 78 años. Igualmente con predominio en sexo femenino $86(60,13 \%)$, pacientes contra y masculinos 57 (39,86\%). Nueve casos estuvieron asociados a esófago de Barrett (ver Figura 4), con predominio de sexo femenino (5 pacientes), con edades comprendidas entre 56 y 73 años y una media de 64 años.
Cuatro fueron negativos para displasia y cinco en la categoría indefinido para displasia.

En total se diagnosticaron dieciocho casos (8\%), con esó-

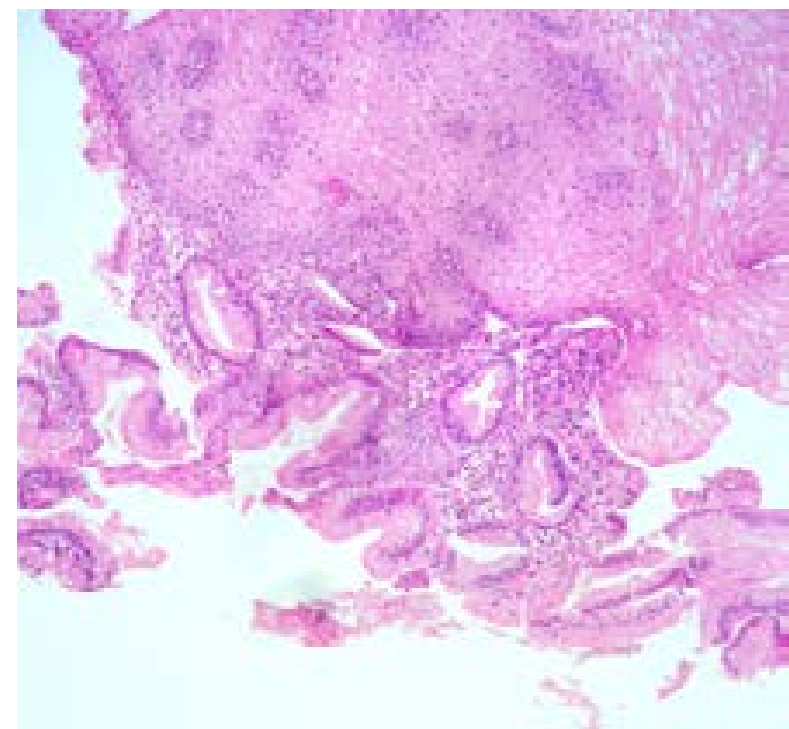

Figura 2. Reflujo asociado a esófago de Barrett. Coloración H-E. 100X

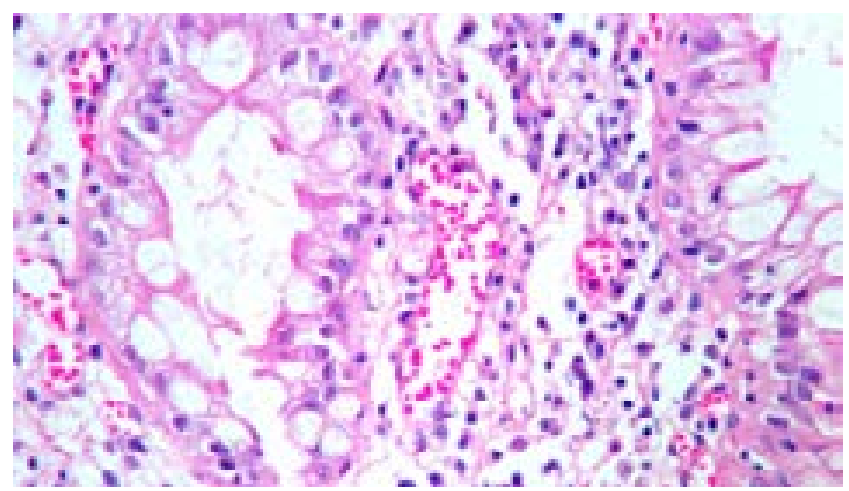

Figura 3. Esófago de Barrett con displasia de bajo grado. Coloración H-E. $400 X$

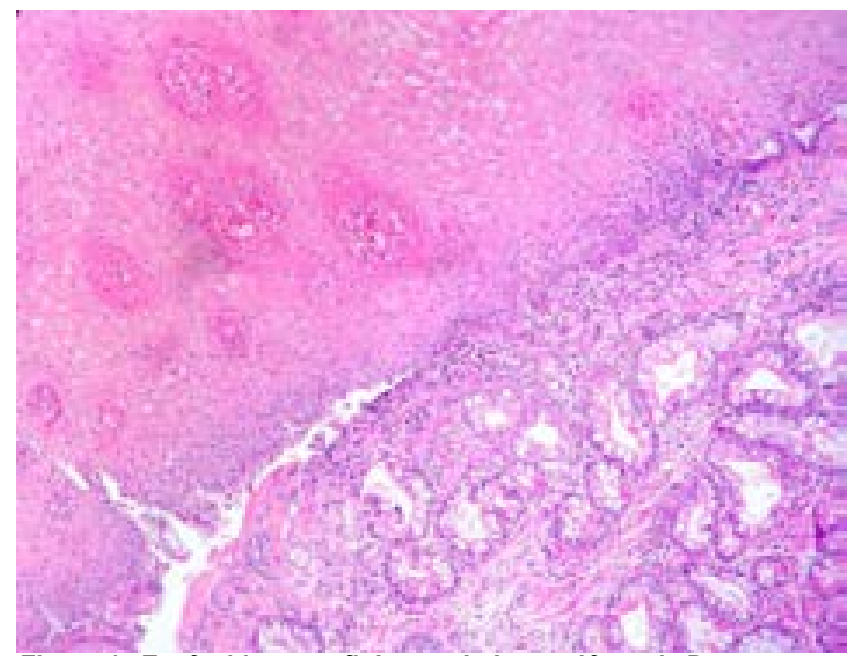

Figura 4. Esofagitis por reflujo asociado a esófago de Barrett. Coloración H-E. 100X 
fago de Barrett en relación a enfermedad por reflujo. Uno con presencia de displasia de bajo grado $(5,55 \%)$, nueve (50\%), como indefinido para displasia y negativo para displasia en 8 casos $(44,44 \%)$. La mayor frecuencia fue en el sexo femenino con $12(66,66 \%)$ pacientes, el masculino presento $6(33,33 \%)$ pacientes, con edades comprendidas entre 30 y 78 años con una media de 59,25 años.

\section{DISCUSIÓN}

Estudios realizados en Norte América, se calcula que el 20 al $25 \%$ de los pacientes presentan enfermedad por reflujo gastroesofágico, sin embargo en el instituto Anatomopatológico de la Universidad Central de Venezuela la frecuencia fue del $31,76 \%$ en diez años de estudio ${ }^{16}$. Un trabajo reciente realizado por Cedeño, et al. obtuvo una prevalencia de esofagitis con hallazgo endoscópico e histológico de 84,37 \% ${ }^{15}$. La enfermedad por reflujo gastroesofágico es una condición común entre los adultos, teniendo una mayor incidencia en mujeres y aumenta con la edad ${ }^{17,18}$. Como lo reporta Poleo en su estudio, donde la prevalencia de enfermedad por reflujo gastroesofágico en el grupo $(A)$ constituido por una población de 337 personas con edad media de 50,9 años, 62,87\% mujeres utilizando elementos clínicos para el diagnóstico y un segundo grupo (B) de 335 personas con edad media de 50, 6 años, 55,49\% mujeres, en el que se estudió la historia clínica, esofagogastroduodenoscopía y biopsia de esófago, resultó en $82,70 \%$ en la no erosiva y erosiva $17,30 \%{ }^{14}$, en el presente estudio predomina el sexo femenino y la edad de presentación es similar. Mientras que en un trabajo publicado en el año 2013, sobre los factores para esofagitis por reflujo y reflujo en 10837 pacientes de Japón, se evidenció que los hombres, desarrollaban con mayor frecuencia esofagitis por reflujo con un porcentaje para este género de $9,8 \%$ y en mujeres 2,4\%; mientras que en los casos de reflujo las mujeres tenían mayor frecuencia presentando valores de $17,1 \%$ y los hombres $15,1 \%^{2}$. A diferencia de nuestros casos donde en ambas patologías el sexo femenino presentó mayor prevalencia.

El esófago de Barrett, está asociado con el reflujo gastroesofágico continuo y se diagnostica en un 10 a $15 \%$ de los pacientes con esofagitis por reflujo ${ }^{17,19}$. Sin embargo en otro estudio se evidenció que el esófago de Barrett estaba asociado a enfermedad por reflujo gastroesofágico de manera general, un 6 a $12 \%$ de los casos, lo cual es concordante con nuestro trabajo ya que se identificó el número de casos asociados de enfermedad por reflujo con Barrett, los cuales fueron 18 casos de 223 en total, lo que correspondió a una prevalencia del $8 \%{ }^{17}$.

En el estudio realizado en nuestra institución entre los años 1995-1999, donde se seleccionaron 266 casos de esofagitis por reflujo y esófago de Barrett, se obtuvo que la edad promedio de los pacientes con esófago de Barrett era 54 años con mayor incidencia en el sexo masculino (62\%) y el $66,7 \%$ fueron negativos para displasia ${ }^{13}$. A diferencia de este estudio donde, el sexo predominante fue el femenino con 12 casos, con una edad promedio de 59 años y la categoría de indefinido para displasia predomino en un $50 \%$ de los pacientes.

Los pacientes con esófago de Barrett, sólo una minoría de ellos, desarrolla cáncer (0,5\%). Encontrándose recientemente en aproximadamente el 0,3\% por año ${ }^{19,20}$. Muchas enfermedades gastrointestinales superiores están aumentando en frecuencia y requieren biopsia para su diagnóstico. El estudio endoscópico superior es un método efectivo para el diagnóstico, control y evolución de la enfermedad por reflujo y su progresión a esófago de Barrett. Es importante el seguimiento de los pacientes con enfermedad de reflujo para prevenir complicaciones graves ya que como se ha descrito anteriormente la evolución es a esófago de Barrett, el cual puede desarrollar displasia de bajo grado y posteriormente de alto grado, progresando a adenocarcinoma ${ }^{20,21}$.

Una limitante del trabajo fue a la falta de información clínica endoscópica indispensable para el correcto diagnóstico del esófago de Barrett.

En conclusión; el presente estudio reporta una prevalencia de $8 \%$ de esófago de Barrett asociado a enfermedad por reflujo gastroesofágico en la sección de patología gastrointestinal y hepática "Dr. Pedro Grases" del Instituto Anatomopatológico "Dr. José A O’Daly" de la Universidad Central de Venezuela. La presencia de displasia de bajo grado estuvo presente en el 5,5\%; La mayor frecuencia fue en el sexo femenino con $66,66 \%$, y las edades comprendidas entre 30 y 78 años con una media de 59,25 años.

\section{REFERENCIAS}

1.VakilN, Van Zanten SV, Kahrilas P, Dent J, Jones R; Global Consensus Group: The Montreal definition and classification of gastroesophageal reflux disease: a global evidencebasedconsensus. Am J Gastroenterol [Internet]. 2006 August [Citado jul 2016];101(8):1900-20. Disponible en: https://www.ncbi .nlm.nih. gov/pubmed $/ 16928254$

2.Chihiro M, Takeshi S, Hikaru K, Yu T, Mitsuhiro F, Yoshiki S. Background Factors of Reflux Esophagitis and Non-Erosive Reflux Disease: a cross-sectional study of 10,837 subjects in Japan. Plos one [internet]. Jul 2013 [Citado 10 jul 2016];8(7):e69891.
Disponible en: http:// journals.plos. org/plosone/article? $\mathrm{id}=10.1371$ /journal. pone.0069891

3.Rui- Hua W. From reflux esophagitis to Barrett's esophagus and esophageal adenocarcinoma. World $J$ Gastroenterol [internet]. May 2015 [Citado 15 jul 2016]; 21(17): 5210-5219. Disponible en: https:// www. wjgnet.com/1 007-9327/ full/v21/i17/5210.htm

4.Rosai and Ackerman. Patología quirúrgica. Tracto gastrointestinal. $10^{\mathrm{a}}$ ed. Nueva York: Amolca; 2011. p. 591.

5.Day D, Jass J, Price A, Shepherd N, Sloan J. Morson and Dawson' s gastrointestinal pathology. $4^{\text {th }}$ ed. USA: Blackwell; 2003. p. 38-43. 6.DenNrdi F, Riddell R. Histología para patólogos. Esófago. $3^{\mathrm{a}}$ ed. Colombia: Amolca; 2007.p. 585. 
7.Taylor J, and Rubenstein JH. Meta-Analyses of the Effect of Symptoms of Gastroesophageal Reflux on the Risk of Barrett's Esophagus. Am J Gastroenterol [Internet].2010 August [Citado 15 jul 2016];105:1730-37. Disponible en: http://www.nature.com/ajg/ journal/v105/n8/full/ajg2010194a.html

8.Turner JR. Tubo Digestivo. En: Kumar V, Abbas A, Fausto N, Mitchell R. Robbins Patología Humana. 8a ed. España:Elsevier; 2010. p. 769-771

9.Cardoza J, Dib J. Esofago de Barrett, Hospital general Dr. Jesus Yerena. Revista Gen. 2013;67(1):42-48

10.González N, Parra-Blanco A, Cohen H. Esófago de Barrett. Acta Gastroenterológica Latinoamericana. Diciembre 2012;42(4).

11.Lacobuzio C, Montgomery E. Patología Hepática y Gastrointestinal. 2da ed: Elsevier;2012.

12. Tamayo-de la Cuesta JL. De la esofagitis al esófago de Barrett. Revista Gastroenterol Mex. 2005.70(1)

13.García M. Estudio clínico morfológico de esófago de barrett en biopsias de pacientes con esofagitis por reflujo 2001[Tesis]. Caracas: Universidad Central de Venezuela;2001.

14.Poleo J. La enfermedad por reflujo gastroesofágico: su prevalencia en dos muestras de población venezolana con referencia a la presencia de alteraciones endoscópicas y al valor de la biopsia esofágica en el diagnóstico de esta condición. Gen 2010;64(3).

15.Cedeño F, Castillo R, González H, Weber W, Hinestroza H, Wagner V. Esofagitis por reflujo diagnóstico histológico y su relación con variables clínicas y endoscópicas. Revista Gen [Internet]. octubre-diciembre 2011;65(4):310-312. Disponible en: http://www.genrevista.org/index.php/GEN/article/view/304/pdf

16. Moore M, Afaneh C, Benhuri Dl, Antonacci C, Abelson J, Zarnegar R. Gastroesophageal reflux disease: a review of surgical decision making. World J Gastrointest Surg [Internet]. Ene 2016 [Citado 16 jul 2016];8(1):77-83. Disponible en: https://www.wjgnet. com/1948-9366/coretip/v8/i1/77.htm

17.Daniele DO, O'Donnell FL, Clark LL. Incidence of gastroesophageal reflux disease (GERD), active component, U.S. Armed Forces, 2005-2014. MSMR [Internet]. Jul 2015 [Citado 20 jul 2016];22(7):14-7. Disponible en: https://www.ncbi.nlm.nih.gov/ pubmed/26207411

18.Dickman R, MAradey-Romero C, Gingold-Belfer R, Fass R. Unmet Needs in the Treatment of Gastroesophageal Reflux Disease. J Neurogastroenterol Motil [Internet]. Jul 2015 [Citado 25 jul 2016];21(3):309-319. Disponible en: https://www.e-sciencecentral. org/articles/SC000011071

19.Bartlesman JF1, Hameeteman W, Tÿtgat GN. Barrett's oesophagus. Eur J Cancer Prev [Internet]. Jun 1992 [Citado 28 jul 2016];1(4):323-5. Disponible en: https://www.ncbi.nlm.nih.gov/ pubmed/1467782

20.Martinucci I, Bartoli N, Russo S, Bertani L, Furnari M, Mokrowiecka A. Barrett's esofhagous in 2016: from pathophysiology to treatment. World J Gastrointest Pharmacol Ther [Internet]. Mayo 2016 [Citado 27 jul 2016];7(2):190-206. doi: 10.4292/wjgpt.v7.i2.190. PMID:27158534; PMCID:pmc4848241. 21.Walker MM, Harris AK, Edwards GC, Talley NJ. A GP primer for understanding upper gastrointestinal tract biopsy reports. Aust Fam Physician[Internet]. Oct 2015 [Citado 27 jul 2016];44(10):70611. Disponible en: https://www.ncbi.nlm.nih.gov/pubmed/26484483 